\title{
COMPETENCIA INVESTIGADORA E INVESTIGACIÓN FORMATIVA EN LA FORMACIÓN INICIAL DEL DOCENTE
}

\author{
Marta Sabariego Puig \\ Ana Belen Cano Hila \\ Begoña Gros Salvat \\ Begoña Piqué Simón \\ Universidad de Barcelona
}

\begin{abstract}
RESUMEN: El presente trabajo es fruto de un proyecto de investigación orientado a desarrollar la competencia investigadora en los estudiantes del Grado de Maestro de Educación Infantil de la Universidad de Barcelona $(U B)^{1}$. Describe la experiencia innovadora desarrollada en entornos formativos duales, a través de la investigación formativa con 46 estudiantes de primer curso del Grado de Maestro de Educación Infantil de la UB. Desde las evidencias obtenidas de la sistematización de esta experiencia, se detalla el diseño del proceso y sus fases, los contenidos trabajados y los resultados obtenidos en el aprendizaje de los estudiantes. Se confirma el desarrollo de habilidades de investigación asociadas a la formación inicial del docente como la autonomía, la búsqueda de información y la gestión de proyectos de investigación para responder a las situaciones problemáticas identificadas. También el desarrollo de competencias de tipo transversal, como el pensamiento crítico y el trabajo en equipo, y las competencias para la transferencia y la comunicación del conocimiento.
\end{abstract}

PALABRAS CLAVE: Enseñanza superior, formación de profesores, formación inicial, práctica pedagógica, relación teoría-práctica.

1. Corresponde al proyecto concedido en el marco de la convocatoria ARMIF2015 -Ajuts de Recerca per a la millora en la Formació Inicial de Mestres- (Núm. Proyecto 00037 ARMIF 2015) titulado "Desenvolupament de competències de recerca a través de la participació en projectes d'aprenentatge servei: del mestre/a reflexiu al mestre/a que investiga". El equipo investigador estuvo formado por A. Ayuste, C. Bovill, A. B. Cano, A. Escofet, B. Gros, Ch. Jouannet, M. Masgrau, M. Payá (investigadora principal), B. Piqué, L. Rubio, M. Sabariego, A. Comas (Escuela La Maquinista, Barcelona), J. N. Pérez (Escuela Corazón de María-Sabastida, Barcelona), Ma T. Sansalvador (Escuela La Farigola de Vallcarca, Barcelona). 


\title{
RESEARCH COMPETENCE AND FORMATIVE RESEARCH IN PRESERVICE TEACHER EDUCATION
}

\begin{abstract}
The present work is the result of a research project with the purpose of developing the research competence in the students of the Early Childhood Degree of the University of Barcelona (UB). This article describes the innovative experience developed in dual training environments, through formative research with 46 students in the Early Childhood Education Degree of the University of Barcelona. It details the design process and its phases, the contents worked, and the results obtained in the students' learning process. It confirms the development of research skills associated with initial training such as autonomy, search for information and creativity in project research to respond to identified problem situations; also, the development of crossdisciplinary skills, critical thinking and teamwork, as well as skills for the transfer and communication of knowledge.
\end{abstract}

KEYWORDS: Higher education, preservice teacher education, teacher education, teaching practice, theory-practice relationship.

Recibido: $31 / 07 / 2019$

Aceptado: 12/05/2020

Correspondencia: Marta Sabariego Puig, Facultad de Educación, Universitat de BarceIona, Campus Mundet, Edifici de Llevant Passeig de la Vall d'Hebron, 171, 2a planta, despatx 276. Barcelona. Email: msabariego@ub.edu.

\section{INTRODUCCIÓN}

La formación teórica y significativa en los grados de educación requiere del vínculo con la actividad de los profesionales en ejercicio, así como la reflexión e implicación de los y las futuras maestras en sus prácticas diarias- complejas, holísticas y socialmente situadas. La formación en y para la investigación también es una necesidad sentida del profesorado en formación inicial (Freixa, 2017) que requiere de este mismo planteamiento didáctico para acercar la investigación educativa y la práctica docente (Vanderlinde y Van Braak, 2010) en pro de una mayor calidad de la educación y la profesionalización de la enseñanza.

Si bien es cierto que este debate es antiguo (Van Manen, 1975; Gauthier, 1984; Zabalza, 1986; Porlan, 1987; Schön, 1987; Liston y Zeichner, 1990) nos sitúa ante un reto aún de plena vigencia: el análisis y la comprensión de los contextos de aprendizaje y las situaciones educativas diarias requieren de una formación específica para definir los problemas detectados, analizar la información, documentar evidencias y comunicar los resultados obtenidos (Colén y Castro, 2017). Exigen desarrollar un estilo profesional basado en la investigación y comprometido con la indagación educativa (Zabalza, 2012) y sitúan la competencia investigadora en el centro del perfil 
profesional del futuro docente para operar en contextos de aprendizaje que vinculen teoría y práctica, desde la deliberación, la reflexión y la investigación (Martínez y Albaigés, 2012; Freixa, 2017; Cano, Pons y Lluch, 2018).

La competencia investigadora incluye elementos conceptuales y técnicos y, sobre todo, aspectos que tienen que ver con las actitudes para encarar la actuación docente, no como simples reproductores o transmisores de conocimientos, sino como personas capaces de crear conocimiento, de investigar sobre su práctica. En palabras de Tejada (2009: 12) "el docente, desde esta perspectiva, será un profesional que ha firmado un compromiso ineludible con el cambio; es decir, es un actor en el escenario de las innovaciones". Hablamos, por tanto, de enseñar para investigar y no tanto de enseñar para hacer investigación científica; de fomentar la competencia investigadora orientada a mejorar la práctica docente. La investigación tiene un significativo valor profesionalizador en la tarea del docente, fomentando, por un lado, la producción intelectual desde la práctica (Astika, 2014) y, por otro lado, la reflexión sobre la práctica, sobre su sentido:

Emerge en este sentido la investigación bajo un carácter de función sustantiva y como tal, en el quehacer del profesor procura la calidad de los procesos educativos y la búsqueda del desarrollo profesional y la práctica del profesor con carácter científico y crítico, de modo que los procesos educativos institucionales sean una respuesta a las necesidades y a los proyectos de desarrollo cultural y científico más allá de la institución escolar (Silva, 2012, 49).

Tack y Vanderlinde (2014) sostienen que es importante formar para enfrentarse a problemas y situaciones que no podemos prever y, por ello, la competencia de indagación e investigación es muy importante. La investigación, según estos autores, ha de ser fruto de la colaboración entre las universidades y los maestros y las maestras en ejercicio. En el proceso de creación de conocimiento basado en la práctica no sólo se genera conocimiento práctico relevante, sino que también se facilita la transferencia del conocimiento. Bronkhorst, Meijer, Koster, Akkerman y Vermunt (2013) descubrieron que, en comparación con otros diseños de investigación, la colaboración basada en la experiencia de la investigación como una parte integrada de la práctica diaria, beneficia de forma clara la formación del profesorado.

La formación del docente como investigador centra el debate más actual entre el enfoque teórico-disciplinar y el enfoque competencial-profesionalizador (Ponte, 2012). Desde el primero, se entiende que el desarrollo de la competencia investigadora requiere la inclusión de los fundamentos y las metodologías de investigación en el plan de estudios, configurándose así un modo de enciclopedismo profesional y disciplinar. El segundo promueve la reflexión e investigación de los docentes desde, en y sobre la práctica docente: el profesorado aprende a partir de su actividad y la reflexión en torno a ella. Las modalidades del aprendizaje en contextos duales universidad-escuela apuestan por esta vía de la formación en la práctica (Martínez y Márquez, 2014; Moreno, 2005; Machado, Montes de Oca, y Mena, 2008; Tack y Vanderlinde, 2014) para el desarrollo de habilidades de investigación, como media- 
ción necesaria para internalizar estructuras de pensamiento y acción que permitan resolver problemas y, en general, para lograr mejores desempeños en la vida cotidiana (Moreno, 2005). En palabras de Ponte (2012):

La investigación, pensada como la forma por excelencia de construcción de conocimiento, cuando está orientada a los problemas de la práctica profesional puede ayudar a identificar estrategias de resolución de estos problemas y asumir un efecto formativo de gran alcance sobre los respectivos participantes. (p. 94).

Este enfoque es el que orienta el proyecto bajo el cual se presenta este trabajo. Su desarrollo ha supuesto el diseño de contextos de investigación en escuelas colaboradoras con la Universidad que permitieran vincular las necesidades de éstas y el desarrollo de las competencias profesionales de los estudiantes. Con este propósito se ha apostado por modalidades de aprendizaje centrado en el estudio y trabajo autónomo (De Miguel, 2006) para el desarrollo de la competencia investigadora a través del codiseño (Rowland, 2008), el Aprendizaje-Servicio (ApS) (Puig, Batlle, Bosch, y Palos, 2006) y la investigación formativa (Restrepo, 2003; Parra, 2004).

El codiseño da cobertura al proceso de construcción didáctica compartida y de participación activa de estudiantes (Bovill y Bulley, 2011). Se desarrolla en ciclos iterativos y continuos de reflexión-acción que se retroalimentan; y vincula procesos de discusión y diseño de dispositivos didácticos (actividades, procesos de evaluación, etc.), aplicación, análisis de resultados y construcción de conocimiento (Rowland, 2008).

Por su parte, el aprendizaje servicio responde a una metodología muy interesante para la docencia inductiva en la educación superior en la medida que "combina los procesos de aprendizaje y servicio comunitario en un solo proyecto bien articulado en el que los participantes aprenden trabajando en las necesidades reales del medio ambiente con el objetivo de mejorarla" (Puig et al., 2006, 22). La participación de los estudiantes en proyectos de ApS permite el desarrollo de la investigación como actividad formativa para producir conocimientos, transferirlos a la práctica profesional y aprender permanentemente (Eyler, 2002; Furco y Billing, 2002).

En esta contribución se describen y analizan los resultados obtenidos específicamente a través de la investigación formativa, para el desarrollo de la competencia de investigación en una asignatura de formación básica del Grado de Educación Infantil de la Universidad de Barcelona. Desde el enfoque competencial-profesionalizador (Ponte, 2012) los y las estudiantes han aprendido a investigar analizando situaciones problemáticas cercanas a su entorno curricular y profesional futuro (Restrepo, 2003; Herrera, 2013; Velandia, Serrano y Martínez, 2017) y gestionando un proyecto de investigación con una propuesta de intervención educativa ajustada a la realidad investigada.

A continuación, se presenta la experiencia desarrollada en colaboración con dieciocho escuelas, la metodología utilizada, la sistematización de la misma (Jara, 2012; Rivas, Leite y Cortés, 2015) y, finalmente, los resultados obtenidos y su discusión en clave de retos de futuro. 


\section{LA INVESTIGACIÓN FORMATIVA, UNA METODOLOGÍA PARA LA FORMACIÓN EN Y PARA LA INVESTIGACIÓN}

La investigación formativa, denominada también "enseñanza a través de la investigación" o "docencia investigadora" (Parra, 2004), constituye una estrategia pedagógica que, mediante las actividades relacionadas con el proceso de investigación, dota a los estudiantes de las habilidades necesarias para la producción académica y su desarrollo profesional (Cortés, Londoño, Luna, Palacios, y Torres, 2008). En nuestro trabajo resultaba de máximo interés por su potencial para el desarrollo de las habilidades investigativas (Moreno, 2005; Miyahira, 2009) a partir de problemáticas reales detectadas en el contexto profesional de los futuros docentes.

La formación para la investigación, así entendida, ha consistido en operativizar la investigación formativa mediante estrategias de enseñanza-aprendizaje guiadas por tres grandes principios (Parra, 2004):

1. La metodología interrogativa: la pregunta y la duda, equiparable al aprendizaje por descubrimiento guiado. Investigar es, en buena medida, descubrir, enfocar lo conocido buscando facetas no conocidas, hacer nuevas preguntas a lo que, en términos de Schütz (1993) es dado-por-sentado.

2. La no directividad. Se favorece un aprendizaje autónomo, en espacios de reflexión epistemológica y construcción progresiva del conocimiento donde el alumnado es interpelado a desarrollar habilidades cognitivas y metacognitivas sobre qué aprende y cómo hacerlo potenciando la capacidad de autorregulación. En palabras de Restrepo (2003: 12), "[la no directividad en la investigación formativa] se refiere más bien a la reconstrucción, organización y reflexión crítica a partir de los problemas mismos que generaron la producción del conocimiento".

3. La docencia inductiva. La formación docente debe darse desde una perspectiva comunitaria, social, esto es, integrando el ámbito contextual del ejercicio docente. El trabajo directo con la comunidad, los contextos reales de prácticas y los entornos profesionales son referentes idóneos, sino teóricos a lo menos pragmáticos, para una mejor comprensión del conocimiento y ejercicio docente.

En esta experiencia los futuros maestros y maestras recorren el camino de la investigación educativa mediante una praxis continua y sistemática en espacios duales universidad-escuela. El contexto de aprendizaje diseñado consistió en la gestión de un proyecto de investigación con una propuesta de intervención educativa ajustada a la realidad investigada para dar respuesta a situaciones problemáticas identificadas en centros reales. Fortalecer el vínculo entre la práctica profesional y la formación para la investigación supuso un reto importante que implicó la reestructuración de la metodología docente en una asignatura de formación básica del Grado de Educación Infantil de la Universidad de Barcelona. 


\section{LA INVESTIGACIÓN FORMATIVA EN LA ASIGNATURA “INTERVENCIÓN EN EL AULA DE EDUCACIÓN INFANTIL"}

"Intervención en el aula de educación infantil" es una asignatura de formación básica dirigida a estudiantes de primero del Grado de Educación Infantil de la Universidad de Barcelona y reconocida con 6 créditos ECTS. La experiencia se desarrolló durante el curso académico 2016-17 con la participación de 46 estudiantes (grupo 4A), distribuidos en 10 equipos de trabajo entre 3 y 6 alumnos, y la colaboración de 18 escuelas de educación infantil ${ }^{2}$, de las cuales tres participaban en el programa de ApS de la Universidad de Barcelona.

Atendiendo a las competencias y objetivos establecidos en el plan docente, se decidió plantear a los estudiantes una experiencia que les acercara a la realidad escolar y a sus problemáticas, para descubrir en qué consiste su intervención en el aula y haciendo hincapié en tareas como: la reflexión sobre la práctica docente, la investigación sobre la misma y la capacidad de mejorarla. Los principales objetivos de esta experiencia innovadora fueron:

- Planificar el propio proceso formativo a partir del cuestionamiento de la práctica docente.

- Aprender los procedimientos de investigación para resolver cuestiones relacionadas con los procesos de enseñanza y el aprendizaje.

- Reflexionar sobre la intervención en el aula y diseñar entornos de aprendizaje en contextos de diversidad.

La experiencia se vertebró a través de un proyecto de investigación formativa, en el cual los estudiantes aprendieron investigando. Concretamente, el proceso se inició con la identificación de problemas que requerían revisión o mejora en unos contextos de análisis reales, se investigó sobre ellos, se obtuvieron resultados y, de éstos, se derivaron propuestas de mejora aplicadas al aula.

\section{Metodología y deSARRollo de la eXPerienCia}

La experiencia formativa y el proceso seguido para su sistematización constó de cuatro fases (ver Tabla 1) que se asociaron con las actividades de aprendizaje y las actividades de evaluación desarrolladas en los contextos duales diseñados: se planificaron tutorías por equipos de trabajo en tres momentos de la experiencia (inicial, seguimiento y final) que se complementaron con la coevaluación realizada por los

2. Las escuelas que han participado son: a) El: Mafalda, Rialles (Badalona), El gall i la gallina, Tabalet (Mataró); b) El-EP: Pablo Candel (L'Hospitalet de Llobregat), Vedruna Gràcia, Les Corts, Joan Pelegrí, Enric Granados, Los Sauces (Navarra), Soler de Vilardell (Sant Celoni), La Maquinista, Baró de Viver, Mare de Déu del Carme (El Prat de Llobregat), Antaviana, El Cabrerès (L'esquirol), Cor de Maria-Sabastida, La Farigola de Vallcarca i Betània-Patmos. Los criterios de selección de estas escuelas fueron diversos: la participación en proyectos de ApS, ser centros de trabajo de algunos/as estudiantes y plantear temáticas de interés o motivación particular para ellos/as. Con la finalidad de iniciar el proceso de colaboración, se contactó vía correo electrónico con las escuelas para agradecer su predisposición y compartirles el planteamiento de la actividad. 
propios estudiantes, y con la valoración de las maestras de las escuelas colaboradoras en las fases finales del proceso.

Tabla 1. Fases del proyecto de investigación y aprendizaje

\begin{tabular}{|c|c|c|c|}
\hline Fases & Actividad de aprendizaje & $\begin{array}{l}\text { Actividad de } \\
\text { evaluación }\end{array}$ & Duración \\
\hline 1. Planificación & Guion del proyecto de investigación & Tutoría inicial & 3 semanas \\
\hline 2. Realización & Proyecto de investigación & Tutoría de seguimiento & 7 semanas \\
\hline 3. Presentación & $\begin{array}{l}\text { Presentación del proyecto de in- } \\
\text { vestigación }\end{array}$ & Coevaluación & 3 semanas \\
\hline 4. Difusión & $\begin{array}{l}\text { Informe final del proyecto de in- } \\
\text { vestigación }\end{array}$ & $\begin{array}{l}\text { Tutoría final } \\
\text { Valoración de las } \\
\text { maestras }\end{array}$ & 3 semanas \\
\hline
\end{tabular}

Fuente: Elaboración propia.

A continuación, se detalla brevemente cada una de estas fases de la experiencia de investigación formativa:

\section{Fase 1. Planificación del proyecto de investigación y aprendizaje}

En primer lugar, se solicitó a los estudiantes la planificación de su proyecto con el apoyo de un guion de trabajo que ofrecía las siguientes consignas a completar: i) el nombre de los miembros del equipo de trabajo; ii) el proceso seguido para escoger la pregunta objeto de investigación y aprendizaje (cómo la escogieron y porqué, teniendo en cuenta los objetivos y competencias recogidas en el plan docente de la asignatura así como las necesidades reales planteadas por las escuelas colaboradoras); iii) la pregunta de investigación y el área temática (lo que pretendían investigar y la redacción del objetivo); iv) el contexto de investigación y los criterios de selección; v) la forma en que se compartiría la pregunta con los responsables de dicho contexto; vi) las técnicas de recogida de información y tratamiento de los datos; vii) el cronograma; viii) los resultados; y, ix) las conclusiones (expectativas, transferibilidad y aprendizaje). Esta tarea se desarrolló durante tres semanas, y en su transcurso la profesora responsable de la asignatura realizó la primera tutoría con cada grupo de trabajo.

\section{Fase 2. Realización del proyecto de investigación y aprendizaje}

Esta segunda fase se refiere al trabajo grupal de los equipos de estudiantes para llevar a cabo el proyecto de investigación según la siguiente estructura formal: título, resumen, palabras clave, introducción y contextualización, objetivos, marco teórico, metodología, resultados, conclusiones con propuestas de mejora, bibliografía y anexos. Cada uno de estos apartados está relacionado con el guión del proyecto planificado en la fase precedente. La ejecución de la investigación, así 
como la sistematización, análisis y reflexión de los datos obtenidos se llevó a cabo durante 7 semanas. En este periodo se compaginaron tanto las tareas propiamente de investigación en el contexto de estudio (el análisis de la información recogida y sistematizada) como la realización de una segunda tutoría grupal para resolver dudas, acompañar el proceso, minimizar obstáculos y aportar recursos, si era necesario.

\section{Fase 3. Presentación del proyecto de investigación y aprendizaje}

La presentación del proyecto de investigación y aprendizaje se desarrolló mediante la organización de unos talleres a lo largo de tres semanas. En estos talleres cada grupo de trabajo presentaba su proyecto al resto del grupo clase y al profesorado de las escuelas colaboradoras invitadas, centrándose fundamentalmente en la exposición de los resultados y las propuestas de mejora.

En esta fase, además de compartir los proyectos desarrollados, se encomendó a los estudiantes una coevaluación de dichos proyectos. Así cada equipo era evaluador de otro grupo, debiendo de leer y valorar el proyecto de sus compañeros. Como resultado de esa valoración, debían redactar un feedback, así como comentarios y/o preguntas para dinamizar los talleres presenciales en el aula.

Con la intención de guiar ese proceso de coevaluación, la docente responsable facilitó a los estudiantes una pauta de evaluación, cuya finalidad fue evaluar la competencia investigadora de los futuros maestros, a través de tres dimensiones básicas: la comunicativa, la metodológica y la competencia de generar conocimiento. En la dimensión comunicativa se valoraban aspectos como: la redacción del proyecto de forma estructurada y con rigor formal, así como la exposición oral de éste. También se valoraba el uso de las TIC en el proceso de exposición y presentación del proyecto de investigación y aprendizaje. En la dimensión metodológica se focalizaba la atención en la delimitación de la pregunta de investigación, la concreción de los objetivos, la coherencia entre el tema de estudio y el marco teórico, así como el diseño de instrumentos de recogida de información. Y finalmente, en la dimensión de generar conocimiento se prestaba atención a si los estudiantes consideraban que habían compartido y reformulado su pregunta de investigación y objetivos con los docentes de las escuelas colaboradoras para una mejor transferencia de los resultados.

La elección de la coevaluación como modalidad de evaluación y seguimiento tiene una clara justificación ética, la cual pretende alejarse de prácticas tradicionales, que vinculan la evaluación al control y la medición; para fomentar la evaluación como una experiencia formativa en la que tanto discente como docente aprenden a través de la continua retroalimentación, no sólo relativa a la parte técnica del aprendizaje, sino con respecto a la relación humana y una práctica profesional responsable y reflexiva. En definitiva, y tal como sostienen, por un lado, Santos (2001) la evaluación es comprender y reflexionar, y, por otro lado, Franco (2008) la evaluación en el ámbito educativo ha de ser ética para un desarrollo verdaderamente humano. 


\section{Fase 4. Difusión del proyecto de investigación y aprendizaje}

En esta última fase se solicitó la elaboración de un Informe final ${ }^{3}$. Éste consistía en el resumen del proyecto de investigación y aprendizaje para compartir los resultados y conclusiones con las personas responsables de las escuelas colaboradoras y solicitarles una breve valoración con relación a la relevancia del contenido, la viabilidad de las propuestas de mejora y su satisfacción global. Para realizar esta última fase se destinaron tres semanas, durante las cuales se alternaban tareas como la redacción del informe final, la tercera tutoría grupal y la evaluación del proceso, a partir de la recogida de los indicadores de la pauta de evaluación facilitada, los grupos de discusión con el alumnado y la valoración de los centros colaboradores en la experiencia.

\section{Método}

El transcurso de la experiencia presentada conllevó su sistematización de acuerdo con el modelo de sistematización de experiencias (De Souza, 2008; Jara, 2012) para recoger, ordenar, y dar sentido a los resultados y aprendizajes obtenidos. La sistematización de experiencias es una actividad investigadora que permite construir y explicitar los saberes que han sido o están siendo producidos en una determinada experiencia por diferentes sujetos, mediante el análisis y valoración de acciones.

En este proceso se consideraron tres focos importantes: a) la obtención y el registro sistemático de toda la información generada; b) la interpretación crítica y c) la producción de conocimientos útiles para enriquecer la práctica. La experiencia desarrollada generó procesos individuales y colectivos, dinámicos y complejos, no simplemente acciones o hechos puntuales, dentro de unos contextos sociales con diferentes agentes. Todo ello justificó el diseño y aplicación de técnicas complementarias para el registro sistemático y exhaustivo de la información (Cabrera, 2011) (ver Tabla 2):

a) el análisis del contenido de las diferentes actividades de aprendizaje y evaluación, detalladas en cada una de las fases (guion, proyecto, presentación del proyecto en el taller, e informe elaborado) con la correspondiente retroacción por parte de la profesora, en función de los indicadores de evaluación establecidos.

b) el registro observacional de las tres sesiones de tutoría grupal con los estudiantes (inicial, seguimiento y final).

c) los grupos de discusión con el alumnado, en el marco de cada proyecto de investigación realizado, con la finalidad de valorar la consecución de los objetivos de la experiencia.

d) el análisis de contenido de la pauta de evaluación facilitada para la coevaluación entre los grupos de estudiantes; y,

e) el análisis de contenido de la valoración emitida por parte de las maestras colaboradoras respecto al informe final.

3. Todos los informes finales del Proyecto de investigación y aprendizaje realizados han sido publicados en el documento Investigar per aprendre a intervenir a l'aula d'educació infantil (Piqué, 2017), Universidad de Barcelona, http://hdl.handle.net/2445/118530 
Tabla 2. Relación de las técnicas, fuentes de información y objetivos

\begin{tabular}{|l|l|l|}
\hline \multicolumn{1}{|c|}{ Técnicas } & \multicolumn{1}{|c|}{ Fuentes de información } & \multicolumn{1}{c|}{ Objetivos } \\
\hline Observación & Las tutorías grupales & $\begin{array}{l}\text { Describir el proceso de aprendizaje } \\
\text { desarrollado por los estudiantes }\end{array}$ \\
\hline $\begin{array}{l}\text { Análisis de } \\
\text { contenido }\end{array}$ & $\begin{array}{l}\text { Actividades de aprendizaje y evalua- } \\
\text { ción (guion, proyecto, presentación } \\
\text { taller, informe presentado) } \\
\text { Retroacción por parte de la profesora } \\
\text { Pauta de evaluación y coevaluación } \\
\text { Valoración de las maestras respecto } \\
\text { al informe final }\end{array}$ & $\begin{array}{l}\text { Sistematizar la información obteni- } \\
\text { do sobre el proceso desarrollado por } \\
\text { parte del alumnado y profesorado } \\
\text { implicado en la experiencia }\end{array}$ \\
\hline $\begin{array}{l}\text { Grupos de } \\
\text { discusión }\end{array}$ & Alumnado participante & $\begin{array}{l}\text { Valorar la consecución de los objeti- } \\
\text { vos de la experiencia }\end{array}$ \\
\hline
\end{tabular}

Fuente: Elaboración propia.

La sistematización es un proceso esencialmente participativo que involucra a las personas relacionadas con la experiencia, y genera un conocimiento social mediante la triangulación de testimonios, para aprender e intervenir desde y para la práctica. La información obtenida con las técnicas anteriores se procesó en un análisis cualitativo (Rodríguez, Lorenzo y Herrera, 2005), dividido en tres niveles progresivos de reducción y estructuración teórica de la información, posteriormente a la transcripción literal de los registros obtenidos: a) la identificación de unidades de análisis relevantes, coherentes con los objetivos del proyecto; b) la organización conceptual de los datos, valorando las fortalezas y debilidades de la experiencia desde la perspectiva del alumnado y desde la perspectiva de las maestras; y c) la presentación de los resultados con respecto a dos aspectos fundamentales: los objetivos y el proceso desarrollado.

Su interpretación, a la luz del marco teórico, derivó en las conclusiones que se presentan al final del artículo con el propósito de ser de utilidad y transferencia para la innovación y mejora del desarrollo de la competencia investigadora en la educación superior.

\section{Resultados}

El análisis realizado requirió la triangulación de las diferentes fuentes de información, contrastando los datos obtenidos y procediendo a su interpretación a partir de tres unidades de análisis relevantes: a) qué supuso para los estudiantes la realización del proyecto de aprendizaje e investigación; b) qué competencias, tareas y acciones necesitaron para lograrlo; y c) qué aprendieron en general sobre educación e investigación en los centros educativos y en la asignatura.

Estas unidades analíticas permitieron organizar conceptualmente la información sobre la competencia investigadora, en dos dimensiones clave para presentar los resultados: las habilidades investigadoras específicamente desarrolladas en el marco de esta experiencia, y el valor de los contextos duales y el proceso llevado a cabo en el transcurso de la experiencia con este propósito. 
A continuación, presentamos las evidencias obtenidas, considerando los objetivos del proyecto y el proceso realizado en el marco de la asignatura, triangulando las fuentes y técnicas anteriores.

\section{Resultados relativos a los objetivos}

La siguiente tabla (tabla 3) ilustra los principales significados contenidos en las transcripciones de los diez grupos de discusión con los estudiantes, categorizadas a partir de las unidades de análisis para evaluar el grado de desarrollo de la competencia investigadora.

Tabla 3. El desarrollo de la competencia investigadora

\begin{tabular}{|l|l|}
\hline \multicolumn{1}{|c|}{ Unidades de análisis } & \multicolumn{1}{c|}{ Competencias de investigación ${ }^{4}$} \\
\hline $\begin{array}{l}\text { Realización del proyecto de } \\
\text { investigación }\end{array}$ & $\begin{array}{l}\text { Esfuerzo }-4 \\
\text { Autonomía }-6 \\
\text { Organización con el grupo }-8 \\
\text { Oportunidad de hacer práctica }-2 \\
\text { Dificultades con el centro educativo }-3\end{array}$ \\
\hline \multirow{2}{*}{$\begin{array}{l}\text { Competencias, tareas y } \\
\text { acciones que se necesitan } \\
\text { Seguir procedimientos }-7 \\
\text { Planificación }-8\end{array}$} \\
$\begin{array}{l}\text { Pensar críticamente }-3 \\
\text { Intervenir de forma adecuada y pertinente }-2 \\
\text { Flexibilidad }-2 \\
\text { Constancia en el trabajo }-3 \\
\text { Trabajo en equipo }-3\end{array}$ \\
\hline Aprendizaje obtenido & $\begin{array}{l}\text { Establecer prioridades }-2 \\
\text { Adaptar la teoría a la práctica }-5 \\
\text { Adaptarse a las necesidades del entorno }-9 \\
\text { Involucrarse en la vida integral del alumno }-6\end{array}$ \\
\hline
\end{tabular}

Fuente: Elaboración propia.

Se evidencia el logro de tres grandes tipos de habilidades de investigación, siguiendo la tipología de Moreno (2005) en la experiencia desarrollada: habilidades instrumentales (establecer prioridades, intervenir de forma adecuada, adaptarse a las necesidades del entorno); habilidades de construcción metodológica (seguir procedimientos, planificación, y búsqueda de información adecuada); finalmente también ha fomentado el desarrollo de habilidades de construcción conceptual y social del conocimiento, para adaptar la teoría a la práctica, pensar críticamente y hacer práctica.

Con respecto al primer objetivo, decidir y planificar el propio proceso formativo a partir del cuestionamiento de la práctica docente, los estudiantes destacan

4. El modo en que se determinó consistió en leer todos los fragmentos correspondientes a cada unidad de análisis y extraer las ideas expresadas con relación al tópico que definía estas unidades de análisis. La cifra indica el número de veces que se repite cada significado referido en los datos analizados. 
dos aportaciones principales de la experiencia: la autonomía y el trabajo en equipo. Afirmaron que la posibilidad de tener autonomía para decidir el objeto de estudio era un proceso enriquecedor e interesante y que les permitía involucrarse más en la asignatura, aunque les requería un mayor esfuerzo, compromiso, constancia, responsabilidad y dedicación. En este sentido, Lucía ${ }^{5}$ y Marta afirman:

"[en relación al planteamiento del proyecto] ha supuesto involucrarme en la asignatura, con el grupo mucha constancia y con la escuela mucha responsabilidad".

"He aprendido a escuchar a los compañeros y ha significado una oportunidad para resolver dudas y asumir retos"

En lo que respecta al trabajo en equipo valoraron la necesidad de saber organizarse, estructurar el trabajo y gestionar el tiempo, pero también la importancia de la cooperación, de saber escuchar a los compañeros, de escoger la pregunta y decidir qué investigar, de discutir y afrontar los problemas, así como de saber interpretar lo que les querían transmitir los maestros.

Con relación al segundo objetivo, aprender los procedimientos de investigación para resolver cuestiones relacionadas con los procesos de enseñanza y el aprendizaje, se destacan tres procedimientos clave para llevar a cabo una investigación: la planificación, la organización y las fuentes de información. En el proceso de planificación manifestaron la necesidad de tener claro lo que se quiere investigar, saber focalizar el tema, y centrarse en pocos objetivos. En cuanto a la organización dieron importancia al hecho de ser constantes, de tener una base sobre cómo investigar, contar con una guía para hacer una investigación práctica, o incluso saber pedir ayuda. En palabras de Martín:

"El aprendizaje de la investigación se mejora con la práctica ya que permite descubrir recursos y métodos para ser más ágiles y obtener mejores resultados"

Y Rosa añade:

"Estar actualizados para hacer investigaciones relevantes"

Respecto a las fuentes de información, valoraron la búsqueda de diferentes fuentes, tanto de libros académicos como de número de escuelas, así como la necesidad de contrastar la información y saber escoger los recursos más adecuados, además de saber interpretar la información y saber escribirla. Alba comenta:

"Enriquecerme de conocimientos para investigar bien, interpretar y saber escribir correctamente lo que quiero decir"

Finalmente, referente al tercer objetivo, reflexionar sobre la intervención en el aula y diseñar entornos de aprendizaje en contextos de diversidad, los estudiantes destacaron la relevancia de la investigación para la innovación educativa y la necesidad de adaptar la intervención en el aula a las características y necesidades del alumnado. En este sentido, manifestaron la dificultad y la exigencia de la investigación, pero al mismo tiempo valoraron la necesidad de investigar para innovar en el ámbito de la educación

5. Los nombres de los estudiantes son ficticios, con el fin de preservar su anonimato. 
infantil y la educación en general, atendiendo a que la educación requiere flexibilidad y adaptabilidad, y que la escuela es una organización compleja donde las situaciones no son "blanco o negro" sino que depende de los objetivos educativos, del trabajo en equipo y del papel de las familias, entre otros. Respecto a esta cuestión, María afirma:

"Es importante ver en qué punto se encuentra la educación de hoy día y observar qué caminos debemos emprender"

Y Diana reafirma:

"Los maestros nunca dejan de aprender".

\section{Resultados relativos al proceso desarrollado}

El proceso de la experiencia se analizó teniendo en cuenta dos niveles (ver Tabla 4): i) interno, centrado en las impresiones de la profesora responsable de la asignatura y el alumnado participante de la experiencia ${ }^{6} ; y$, ii) externo, focalizado en la relación de la experiencia planteada con las escuelas y maestras colaboradoras ${ }^{7}$.

Tabla 4. Síntesis de los resultados sobre el proceso del proyecto de Investigación y Aprendizaje en la asignatura Intervención en el aula de educación infantil

\begin{tabular}{|c|c|c|}
\hline & Aspectos favorables & Aspectos desfavorables \\
\hline $\begin{array}{l}\text { Nivel interno } \\
\text { (profesora, } \\
\text { alumnado y } \\
\text { proyecto) }\end{array}$ & $\begin{array}{l}\text { Planificación proceso } \\
\text { Autonomía de trabajo } \\
\text { Nivel de } \\
\text { autoexigencia } \\
\text { Acompañamiento } \\
\text { profesora en el } \\
\text { proceso }\end{array}$ & $\begin{array}{l}\text { Desconcierto inicial alumnado } \\
\text { Poca implicación de los alumnos } \\
\text { Prevalencia del mínimo esfuerzo } \\
\text { Cierta resistencia a afrontar una tarea sobre la } \\
\text { que no tenían una experiencia previa } \\
\text { Dificultades para reconducir la tarea a partir de } \\
\text { la retroacción de la profesora } \\
\text { Escasas estrategias de superación de las dificultades } \\
\text { implícitas en cualquier proceso de investigación }\end{array}$ \\
\hline $\begin{array}{l}\text { Nivel externo } \\
\text { (relación del } \\
\text { proyecto con las } \\
\text { escuelas y maestros } \\
\text { y maestras } \\
\text { colaboradoras) }\end{array}$ & $\begin{array}{l}\text { La disponibilidad de } \\
\text { las escuelas } \\
\text { La implicación de las } \\
\text { maestras para atender } \\
\text { a los estudiantes }\end{array}$ & $\begin{array}{l}\text { Diferencia de tiempos entre la planificación de } \\
\text { la asignatura en la universidad (semestre) y el } \\
\text { calendario de las escuelas (trimestre) } \\
\text { Dificultades en la sincronización de ritmos de } \\
\text { trabajo } \\
\text { Problemas de comunicación entre partes im- } \\
\text { plicadas } \\
\text { Expectativas creadas sobre el resultado y el im- } \\
\text { pacto del proyecto por parte de cada uno de } \\
\text { los actores implicados en la experiencia } \\
\text { Cierta decepción o desencanto ante los resul- } \\
\text { tados obtenidos }\end{array}$ \\
\hline
\end{tabular}

Fuente: Elaboración propia.

6. Datos obtenidos del registro de los grupos de discusión con el alumnado y la profesora de la asignatura.

7. Esta información ha sido obtenida a partir del análisis de contenido de la valoración emitida por parte de las maestras colaboradoras respecto al informe final. 
En el nivel interno, la profesora y los y las estudiantes valoraron favorablemente la planificación de todo el proceso (calendario, equipos de trabajo, tareas); la autonomía en el trabajo (decisión de la pregunta, elección de las escuelas, búsqueda de información, visitas a las escuelas); el nivel de autoexigencia (superación de obstáculos, implicación progresiva y apertura a los cambios); la relación de confianza entre profesora y estudiantes; y, la implicación de ésta en el proceso de investigación (acompañamiento y apoyo a los estudiantes en la toma de conciencia de su formación inicial y la valoración realista del informe final).

Por contra, señalaron como aspectos menos favorables los relativos al desconcierto inicial de los estudiantes ante el planteamiento novedoso de la asignatura. Al principio, éste generó poca implicación, la prevalencia del mínimo esfuerzo y ciertas resistencias a afrontar las dificultades implícitas en cualquier proceso de investigación: por ejemplo, la elaboración de la pregunta, la identificación de las variables de estudio, la utilización de fuentes de información más rigurosas, la elaboración de los instrumentos de obtención de información coherentes con las variables de estudio, o la explicitación del análisis de los resultados, entre las más relevantes. Desde la mirada de los estudiantes, este desconcierto se tradujo en cierta inseguridad, tanto con respecto a sus conocimientos como sus competencias (dudas de saber hacer, sentimiento de pérdida y falta de expectativas clara en cuanto a lo que se exigía).

En el plano más externo y relativo al trabajo con los centros, se valoró positivamente la disponibilidad de las escuelas y la implicación de las maestras para atender a los estudiantes (tiempo de dedicación y poner al alcance de los estudiantes toda la información disponible).

Por otra parte, los aspectos desfavorables surgieron, fundamentalmente, de la diferencia de tiempos entre la planificación de la asignatura en la universidad (semestre) y el calendario de las escuelas (trimestre). Este desajuste obstaculizó la sincronización de ritmos de trabajo, así como el desarrollo completo del proyecto o la experiencia, lo cual en algunas ocasiones derivó en tensiones y problemas de comunicación entre todos los actores implicados (profesorado, alumnos y maestras colaboradoras). También se apreciaron desequilibrios entre las expectativas creadas sobre el resultado y el impacto del proyecto que, en algunos momentos, provocaron cierta decepción. En el caso de los estudiantes, por ejemplo, pretendían alcanzar resultados muy relevantes que conllevaran un cambio significativo en el centro; pero en realidad vieron que el alcance de esas propuestas fue menor al esperado. $Y$ las maestras colaboradoras, por su parte, esperaban que los estudiantes realizaran una investigación y un proyecto de mejora orientado a sus necesidades reales, lo cual, les permitiera pensar propuestas de innovación educativas factibles. En algunos casos las aportaciones de los estudiantes se alejaron notablemente de sus necesidades y les resultaron poco útiles o valiosas.

En cuanto a la colaboración en contextos duales, tanto desde la perspectiva del alumnado como la perspectiva de las maestras, se manifestaron aquellos elementos fundamentales que permitieron la resolución de problemas y el desarrollo 
de la competencia investigadora y profesional de los estudiantes (ver Tabla 5) ${ }^{8}$ : la principal aportación de la colaboración en estos espacios duales es la oportunidad de dar respuesta a una cuestión planteada (el problema de investigación) para una mejor atención educativa real, ampliar las fuentes de información para contrastarlas y hacerse una opinión propia y compartir el objetivo de la investigación. También es relevante el contacto directo y periódico que favorece para facilitar la comunicación, el contraste de la realidad de la escuela para reconocer las necesidades y la adopción de un lenguaje común de trabajo asequible por todos los participantes e implicados.

Tabla 5. Ejemplo de análisis cualitativo

\begin{tabular}{|l|c|c|}
\hline \multicolumn{1}{|c|}{ Colaboración en contextos duales } & $\begin{array}{c}\text { Maestras } \\
\text { (18 escuelas) }\end{array}$ & $\begin{array}{c}\text { Grupos de } \\
\text { estudiantes (10) }\end{array}$ \\
\hline Contacto directo y periódico & 15 & 7 \\
\hline Lenguaje común de trabajo & 8 & 5 \\
\hline Consensuar tiempos & 15 & 7 \\
\hline Contrastar la realidad escolar & 13 & 9 \\
\hline Compartir el objetivo de la investigación & 9 & 6 \\
\hline Ampliar las fuentes de información & 9 & 10 \\
\hline $\begin{array}{l}\text { Mejorar la atención educativa en el curso } \\
\text { escolar }\end{array}$ & 17 & \\
\hline
\end{tabular}

Fuente: Elaboración propia.

La experiencia desarrollada ha permitido introducir la visión de la investigación en los estudiantes, como un método de trabajo para el diagnóstico y la evaluación de la práctica educativa, desde una dimensión individual y sobre todo colectiva, muy próxima a su desarrollo profesional. También ha exigido la necesidad de considerar lo que cada uno/a aporta, a organizarse en función del tiempo disponible, a planificar las actividades y distribuir responsabilidades, a compartir intereses y consensuar conceptos, a concretar los encuentros con las maestras y readaptar los tiempos, en definitiva, a adquirir valores propios del trabajo en equipo.

\section{Discusión Y CONCLUSIONES}

A partir de los resultados obtenidos, concluimos que la investigación formativa permite desarrollar competencias de investigación en la formación inicial de maestros. Se ha evidenciado la necesidad de hacer consciente a los estudiantes, futuros maestros, de la importancia de la competencia investigadora como un método de tra-

8. El modo en que se determinó consistió en leer todos los fragmentos de los registros obtenidos y extraer las ideas o significados expresados con relación a este tópico (la valoración de la colaboración en los contextos duales). La cifra indica el número de veces que estos significados referidos se repiten en los datos. 
bajo para el diagnóstico y la evaluación de la práctica educativa. Este posicionamiento es coherente con los resultados obtenidos en investigaciones previas (Bronkhorst et al., 2013) que muestran la importancia de trabajar la competencia investigadora a partir de la práctica.

La investigación formativa, en tanto práctica pedagógica, ha permitido el logro de tres grandes tipos de habilidades de investigación, siguiendo la tipología de Moreno (2005): habilidades instrumentales (cuestionar la práctica, detectar necesidades y formular problemas de investigación); habilidades de construcción metodológica (identificar las fases principales de un proyecto investigador, y sistematizar procesos como la revisión de la literatura y la documentación sobre un tema, la observación en los contextos reales, y el desarrollo de la obtención y el análisis de los datos obtenidos); finalmente, también ha fomentado el desarrollo de habilidades de construcción conceptual y social del conocimiento en el ejercicio de elaboración de los informes y la difusión de los resultados obtenidos vía talleres.

Desde el plano del desarrollo del proceso de aprendizaje es interesante la valoración de cuatro sub-competencias claves y prioritarias para el desarrollo de una investigación, desde la perspectiva del alumnado: la importancia de la planificación (tener claro qué investigar- el problema y los objetivos); la identificación de las necesidades profesionales y educativas como punto de partida; el valor de la sistematización del proceso (ser constantes, tener una guía o una pauta de qué y cómo hacerlo); y la disposición de fuentes documentales y personales para analizar e interpretar la información.

Estas aportaciones van más allá de los objetivos docentes de la asignatura en la que se contextualiza esta experiencia, y evidencian el potencial de la investigación formativa, en tanto práctica pedagógica, para lograr la formación de competencias cognitivas y metodológicas, el aprendizaje permanente y la generación de nuevo conocimiento:

La investigación formativa es fundamental en la formación para la investigación y para profesionales con pensamiento crítico, con capacidad para el aprendizaje permanente, para la búsqueda de problemas no resueltos y para plantear soluciones en su tarea cotidiana, características del tipo de profesionales que requiere el país" (Hernández, 2003, 122).

Si se atiende al escenario académico de esta experiencia para el desarrollo de la competencia investigadora, se advierten características clave que ya han sido reconocidas previamente (Moreno, 2005): un quehacer académico consistente en promover y facilitar, preferentemente de manera sistematizada (no necesariamente escolarizada), el acceso a los conocimientos, al desarrollo de habilidades, hábitos y actitudes, y a la internalización de los valores que demanda el quehacer investigativo (práctica de la investigación). Una idea que refuerza la visión integral en la formación de investigadores desde la práctica y reconoce la incidencia socioeducativa en el proceso de su desarrollo. Es importante reconocer estos valores en la experiencia presentada: la reflexión desde las experiencias vividas en los centros educativos; el contraste e interacción de propuestas con los maestros e iguales; el aprendizaje autónomo y au- 
torregulado y el vínculo teoría y práctica para desarrollar habilidades de pensamiento de orden superior (utilizar, aplicar, dar sentido) son componentes esenciales de unos contextos que han aportado:

- La mayor exigencia y responsabilidad en el alumnado, desde el desconcierto inicial y ciertas resistencias hasta la valoración positiva del aprendizaje desde la experiencia y el acompañamiento de los maestros en activo (realismo y priorización de necesidades).

- Una mejor comparación de la realidad educativa con los conocimientos científicos y los valores éticos-profesionales.

- El desarrollo del estudio de lo complejo: el contexto en sus interrelaciones y globalidad y la visión integral necesaria para la formación en investigación.

La investigación formativa permite integrar diferentes dimensiones y niveles de formación: la perspectiva teórica, empírica y la dimensión social. Esta experiencia así lo confirma, igual que su potencial para el desarrollo de competencias asociadas a la investigación en la formación inicial (Guerrero, 2007): autonomía, rigor, compromiso y responsabilidad social, gestión de proyectos, argumentación científica y comunicación. Se da cabida a una visión de proceso y de lógica de desarrollo de habilidades (no sólo de contenidos o pasos a seguir) para enseñar a investigar.

Finalmente, la mirada crítica y problematizadora no es espontánea, es necesario tener formación para ser capaz de plantear preguntas de investigación a partir de la observación directa en el aula. Se trata de una habilidad asociada a la realidad educativa y conduce a la identificación de problemas profesionales educativos. Según Martínez y Márquez (2014) implica la percepción de contradicciones en el contexto profesional, mediante la comparación de la realidad educativa con los conocimientos científicos y valores éticos-profesionales que tiene el sujeto. La experiencia desarrollada nos permite dar valor e importancia al papel activo de los estudiantes en la construcción individual y social de estos problemas. Los estudiantes se han planteado cuestiones educativas relacionadas, sobre todo, con su experiencia académica y ciertos tópicos educativos fruto de su experiencia sociolaboral. Conocer la escuela, su realidad, sus intereses y necesidades, requiere un espacio y un tiempo que el desarrollo de la experiencia no ha permitido. Sin embargo, ha sido posible contrastar sus inquietudes con las maestras de las escuelas, proporcionándoles cierto realismo a los problemas planteados y acercándose de este modo a su ejercicio profesional.

En síntesis, con la voluntad de no considerar investigación cualquier hibridación teoría-práctica y depauperar este concepto, creemos que es importante plantear una serie de reflexiones sobre cómo incardinar la competencia investigadora en los estudios de Educación, adecuando la ambición del término al estadio de formación de los futuros y futuras maestras:

- Precisar el procedimiento de trabajo y orientar detallada y ampliamente la elaboración de los proyectos de investigación, a través de una pauta y guía de trabajo. Lo más interesante del enfoque metodológico que ofrece la investigación formativa es que el aprendizaje no sólo se refiere al conocimiento nuevo que 
puede y debe adquirir el alumnado, sino al desarrollo y evolución de su modo y estilo de aprender y mejorar cómo aplicar este conocimiento a situaciones nuevas, cómo integrar las actitudes y valores, cómo ponerlos en juego, y cómo incorporar las técnicas y métodos a la hora de afrontarlas. Se trata de un tipo de aprendizaje que requiere de un proceso de enseñanza con modalidades organizativas centradas en el estudio y trabajo autónomo en la educación superior (De Miguel, 2006), dando así cabida a una visión de proceso y de lógica de desarrollo de habilidades y competencial-profesionalizador (Ponte, 2012) para enseñar a investigar (aprender a formular problemas y proyectos, idear hipótesis, diseñar su propia metodología de investigación, recopilar información, procesar datos, argumentar y presentar resultados).

- Incentivar la participación de los estudiantes en la construcción y regulación del propio proceso de aprendizaje, incorporándose en programas que permitan la contextualización profesional y la gestión de la complejidad en las instituciones educativas, como por ejemplo el Aprendizaje Servicio, o la vinculación a proyectos de investigación dentro de las asignaturas que se estén cursando (por ejemplo, el Trabajo Final de Grado). La intervención continuada en estas actividades permite el pleno desarrollo de la competencia investigadora, reconociendo la incidencia socioeducativa en su proceso de desarrollo a partir de ciclos iterativos que hibridan teoría, práctica y reflexión.

- Propiciar procesos de enseñanza y aprendizaje en contextos formativos duales universidad-escuela o Sociedad, como vía de la formación en la práctica (Martínez y Márquez, 2014; Moreno, 2005; Machado et al., 2008) para el desarrollo de la competencia investigadora. Esto implica planificar y desarrollar un conjunto de actividades relacionadas con el proceso de investigación, no sólo para la formación inicial de los estudiantes sino también para la formación continuada de los profesores. Es necesario compartir las habilidades investigativas de todos los sujetos, noveles y veteranos, a través de acciones orientadas al proceso de "aprender a investigar investigando" que sirvan de apoyo a la práctica profesional.

\section{REFERENCIAS BIBLIOGRÁFICAS}

Astika, G. (2014). Reflective teaching as alternative assessment in teacher education. A case study of preservice teachers. TEFLIN Journal, 25(1), 16-32.

Bovill, C., y Bulley, C. (2011). A model of active student participation in curriculum design: exploring desirability and possibility. En C. Rust (Coord.), Improving Student Learning (ISL): Global Theories and Local Practices: Institutional, Disciplinary and Cultural Variations (pp. 176-188). Oxford: The Oxford Centre for Staff and Educational Development.

Bronkhorst, L. H., Meijer, P. C., Koster, B., Akkerman, S. F. y Vermunt, J. D. (2013). Consequential research designs in research on teacher education. Teaching and Teacher Education, 33, 90-99. https://doi.org/10.1016/j.tate.2013.02.007 
Cabrera, F. A. (2011). Técnicas e instrumentos de evaluación: una propuesta de clasificación. REIRE, Revista d'Innovació i Recerca en Educació, 4(2), 112-124. https:// doi.org/10.1344/reire2011.4.2428

Cano, M. E., Pons, L. y Lluch, L. (2018). Análisis de experiencias de innovación docente universitarias sobre evaluación. Profesorado. Revista de Currículum y Formación de Profesorado, 22(4), 11-32.

Colén, M. T. y Castro, L. (2017). El desarrollo de la relación teoría y práctica en el Grado de Maestro en Educación Primaria. Revista de Currículum y Formación de Profesorado, 21(1), 59-79.

Cortés, J., Londoño, B., Luna, B. E., Palacios, M. T. y Torres, M. L. (2008). Investigación Formativa nuevas propuestas pedagógicas en las Facultades de Derecho. Caso: Facultad de Jurisprudencia de la Universidad del Rosario. Studiositas, 3(34), 28-33.

De Miguel, M. (2006). Modalidades de enseñanza centradas en el desarrollo de competencias. Orientaciones para promover el cambio metodológico en el EEES. Madrid: MEC/Universidad de Oviedo.

De Souza, J. F. (2008). Sistematización: un instrumento pedagógico en los proyectos de desarrollo sustentable. Revista Internacional Magisterio. Educación y Pedagogía, 23, 9-13.

Eyler, J. (2002). Reflection: Linking Service and Learning-Linking Students and Communities. Journal of Social Issues, 58(3), 517-534. https://doi.org/10.1111/15404560.00274

Franco, Z. R. (2008). ¿Por qué la evaluación en el medio educativo debe ser ética para un desarrollo verdaderamente humano? Eleuthera, 2, 144-164.

Freixa, M. (2017). Professorat novell: Competències docents a l'inici de l'exercici professional. Barcelona: Agència per a la Qualitat del Sistema Universitari de Catalunya (AQU).

Furco, A. y Billing, S. (Eds.) (2002). Service-Learning. The essence of the pedagogy. Greenwich (CT): Information Age Publishing.

Gauthier, S. A. (1994). Attitudes toward science and science teaching as reflected in the science autobiographies of preservice elementary teachers. Unpublished doctoral dissertation. New Hampshire: University of New Hampshire.

Guerrero, M. E. (2007). Formación de habilidades para la investigación desde el pregrado. Acta Colombiana de Psicología, 10(2), 190-192.

Hernández, C. (2003). Investigación e investigación formativa. Nómadas, 18, 183-193.

Herrera, J. D. (2013). Pensar la educación, hacer investigación. Bogotá: Universidad de la Salle.

Jara, O. (2012). Sistematización de experiencias, investigación y evaluación: aproximaciones desde tres ángulos. Revista Internacional de Investigación en Educación Global y para el Desarrollo, 1, 56-70. 
Lanuez, M. y Pérez, V. (2005). Habilidades para el trabajo investigativo: experiencias en el Instituto Pedagógico Latinoamericano y Caribeño (IPLAC). La Habana: IPLAC.

Liston, D. P. y Zeichner, K. M. (1990). Reflective teaching and action research in preservice teacher education. Journal of Education for Teaching, 16(3), 235-254.

Machado, E., Montes de Oca, N. y Mena, A. (2008). El desarrollo de habilidades investigativas como objetivo educativo en las condiciones de la universalización de la educación superior. Revista Pedagogía Universitaria, 13(1), 156-180.

Martínez, D. y Márquez, D. L. (2014). Las habilidades investigativas como eje transversal de la formación para la investigación. Tendencias pedagógicas, 24, 347360.

Martínez, M. y Albaigés, B. (2012). L'estat de l'educació a Catalunya. Anuari 2013. Barcelona: Fundació Jaume Bofill.

Mateo, J. (2006). Claves para el diseño de un nuevo marco conceptual para la medición y evaluación educativas. Revista de Investigación Educativa, 24(1), 165-186.

Miyahira, J. (2009). La investigación formativa y la formación para la investigación en el pregrado. Revista Médica Herediana, 20(3), 19-122. https://doi.org/10.20453/ rmh.v20i3.1010

Moreno, M. G. (2005). Potenciar la educación. Un currículum transversal de formación para la investigación. Revista Electrónica Iberoamericana sobre Calidad, Eficacia y Cambio en Educación, 3(1), 520-540.

Parra, C. (2004). Apuntes sobre la investigación formativa. Educación y Educadores, 7, 57-78.

Ponte, J. (2012). Estudiando el conocimiento y el desarrollo profesional del profesorado de matemáticas. En N. Planas (Coord.), Teoría, Crítica y Práctica de la educación matemática (pp. 83-98). Barcelona: Graó.

Porlán, R. (1987). El maestro como investigador en el aula. Investigar para conocer, conocer para enseñar. Revista Investigación en la Escuela, 1, 63-69.

Puig, J. M., Batlle, R., Bosch, C. y Palos, J. (2006). Aprenentatge Servei. Educar per a la ciutadania, Barcelona: Octaedro.

Restrepo, B. (2003). Conceptos y aplicaciones de la Investigación Formativa y criterios para evaluar la investigación científica en sentido estricto. Bogotá: CNA.

Rivas, J., Leite, A. y Cortes, P. (2015). La escuela como contexto de la formación inicial del profesorado: aprendiendo desde la colaboración. Revista de Currículum y Formación de Profesorado, 19(1), 228-242.

Rodríguez Sabiote, C., Lorenzo Quiles, O. y Herrera Torres, L. (2005). Teoría y práctica del análisis de datos cualitativos. Proceso general y criterios de calidad. Revista Internacional de Ciencias Sociales y Humanidades, SOCIOTAM, XV(2), 133-154. 
Rowland, G. (2008). Design and research: Partners for educational innovation, Educational Technology, 48(6), 3-9.

Santos, M. A. (2001). Dime cómo evalúas (en la universidad) y te diré qué tipo de profesional (y de persona) eres, Tendencias pedagógicas, 6, 89-100.

Silva, W. H. (2013). Investigación y práctica reflexiva como categorías epistemológicas del desarrollo profesional docente. Revista de Docencia e Investigación, 27(62), 53-64. https://doi.org/10.21500/01212753.1498

Schön, D. A. (1987). Educating the reflective practitioner. San Franscisco: Jossey-Bass.

Schütz, A. (1993). La construcción significativa del mundo social. Barcelona: Paidós.

Tack, H. y Vanderlinde, R. (2014). Teacher Educators' Professional Development: Towards a Typology of Teacher Educators' Researcherly Disposition. British Journal of Educational Studies, 62(3), 297-315. https://doi.org/10.1080/00071005.20 14.957639

Tejada, J. (2009). Competencias docentes. Profesorado. Revista de currículum y formación del profesorado, 13(2), 1-15. Recuperado de http://www.ugr.es/ recfpro/ rev132COL2.pdf

Vanderlinde, R. y van Braak, J. (2010). The gap between educational research and practice: views of teachers, school leaders, intermediaries and researchers. British Educational Research Journal, 36(2), 299-316. https://doi. org/10.1080/01411920902919257

Van Manen, M. J. (1975). An exploration of alternative research orientations in social education. Theory and Reserch In Social Education, 3(1), 1-28.

Velandia, C., Serrano, F. J. y Martínez, M. J. (2017). La investigación formativa en ambientes ubicuos y virtuales en Educación Superior. Comunicar, XXV(5), 9-18. https://doi.org/10.3916/C51-2017-01

Zabalza, M. A. (1986). El paradigma del pensamiento del profesor y sus aplicaciones a la formación y desarrollo profesional del profesorado. Paper. First International Meeting on Psychological Teacher Education. Braga Mayo.

Zabalza, M. Á. (2012). Las competencias en la formación del profesorado: de la teoría a las propuestas prácticas. Tendencias Pedagógicas, 20, 5-32. 